\title{
Chemoselective, Metal-free, (Hetero)Arene Electroreduction Enabled by Rapid Alternating Polarity
}

\author{
Authors: Kyohei Hayashi ${ }^{1}$, Jeremy Griffin ${ }^{2}$, Kaid C. Harper ${ }^{2}$, Yu Kawamata*1, Phil S. Baran*1
}

\author{
Affiliations: \\ ${ }^{1}$ Department of Chemistry, The Scripps Research Institute, 10550 North Torrey Pines Road, La \\ Jolla, CA 92037, USA. \\ ${ }^{2}$ Abbvie Process Research and Development, 1401 North Sheridan Road, North Chicago, IL, \\ 60064, United States.
}

*Correspondence to: yukawama@scripps.edu, pbaran@scripps.edu

\begin{abstract}
:
Arene semi-reduction remains a challenge when multiple reductively labile functional groups are present or when using heteroarene substrates. Conventional chemical and even electrochemical Birch-type reductions suffer from a lack of chemoselectivity due to a reliance on alkali metals or harshly reducing conditions. This study reveals that a simpler avenue is available for such reductions by simply altering the waveform of current delivery, namely rapid alternating polarity (rAP). The developed method, which proceeds in protic solvent and can be easily scaled up, does not require any metal additives or stringently anhydrous conditions. The scope of this dearomatization is broad, tolerating numerous functional groups and providing rapid access to previously challenging molecules. While the mechanism has not been fully deciphered, the key feature of the rAP reduction is that the main competing process, namely proton reduction, can be suppressed. As such, unique arene reductions can be accomplished even outside solvent electrochemical window or in the presence of Brønsted acids.
\end{abstract}

\section{MAIN TEXT:}

The addition of hydrogen to an aromatic nucleus represents a widely used strategy for rapidly introducing complexity in synthesis. ${ }^{1}$ As such, the impact of the Birch reduction and related dearomatization strategies in organic synthesis cannot be overemphasized. ${ }^{2-5}$ This foundational reaction of organic chemistry, taught at the undergraduate level, is also notorious for its harsh reaction conditions: liquid ammonia, elemental alkali metal $(\mathrm{Li}, \mathrm{Na}, \mathrm{K})$, and a judicially chosen proton source (Figure 1A). ${ }^{2}$ Owing to the hazardous nature of these conditions, the search for more practical variants is still an active research topic in modern organic synthesis 70+ years following its original disclosure. These efforts can be placed into two categories: (1) conditions that ablate inherent safety hazards (elemental alkali metal and liquid ammonia) and (2) improving the chemoselectivity and scope. Within the former category, various solid-supported reagent systems $\mathrm{s}^{6-8}$ have been disclosed as well as mineral oil dispersions with crown-ether additives. ${ }^{9}$ In 2019 a practical electrochemical Birch variant inspired by $\mathrm{Li}$-ion batteries was developed for ammonia and elemental $\mathrm{Li}$-free arene reduction (Li-ion electroreduction, LER). ${ }^{10}$ Photochemical variants have also been described, although the scope and reaction times required for those systems are suboptimal. ${ }^{11,12}$ Recently the Koide group demonstrated that ammonia could be replaced by ethylene diamine (e.g. Benkesser modification ${ }^{13-15}$ ) to further enhance practicality. ${ }^{16}$ Although some of these studies enabled practical and scalable Birch reduction, the chemoselectivity was revealed to be analogous to 
conventional Birch reduction. To improve chemoselectivity, the procedure employing LiDBB reported by Donohoe and co-workers is notable since it is widely used for reduction of electron-deficient heteroarenes with good tolerance of esters, which seldom survives during Birch reduction. ${ }^{17}$ This Communication discloses the finding that simply modifying the waveform of electrolysis (rapid alternating polarity, rAP) can lead to a new level of chemoselectivity for arene and heteroarene reduction. This operationally simple protocol proceeds at ambient temperature in protic solvent, does not require sacrificial electrodes, or even any metal source, and tolerates functional groups that are notoriously challenging to accommodate such as ester, nitrile, epoxide and even alkyl chloride.

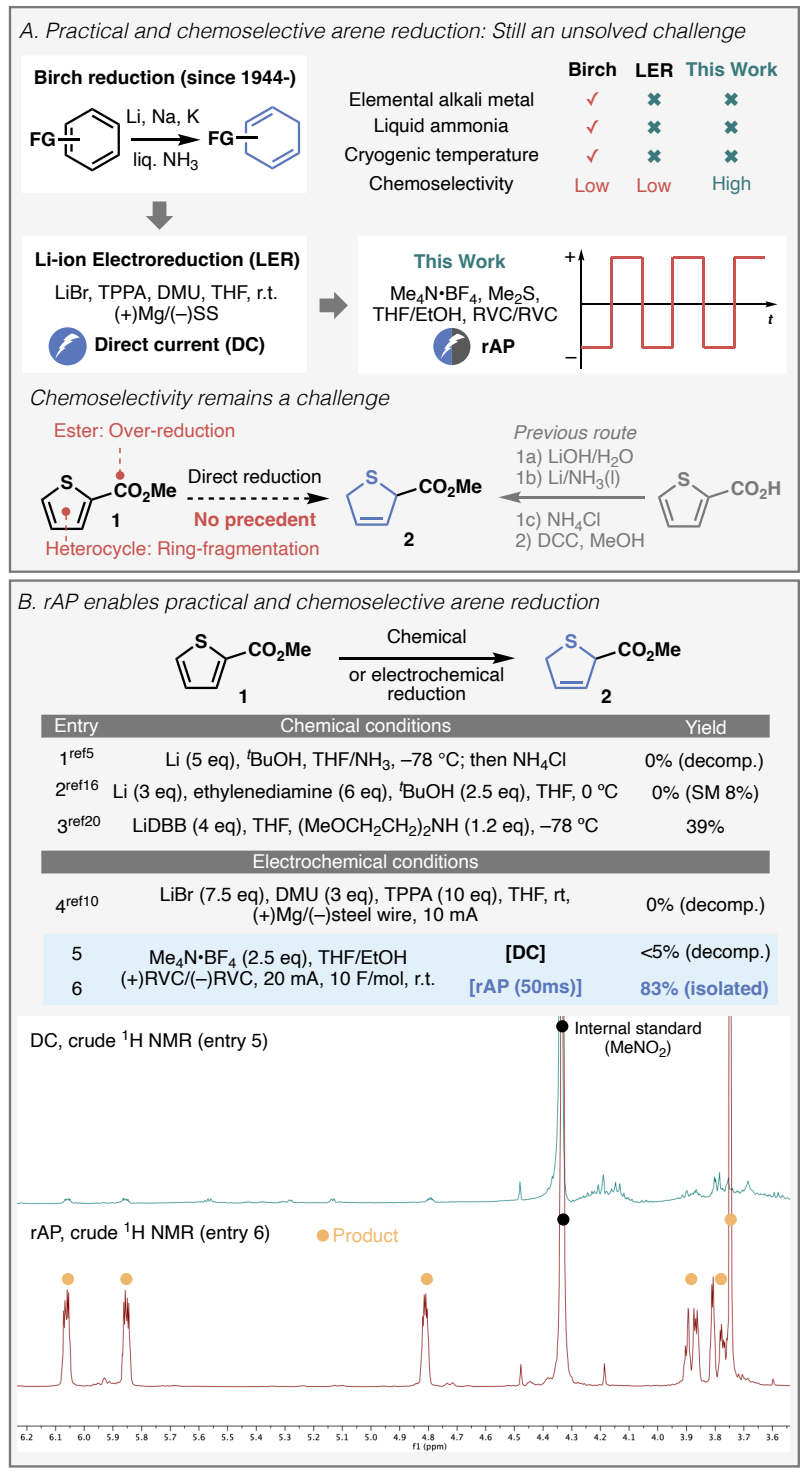

Figure 1. Background of arene reduction and discovery of efficient electroreductive dearomatization by rAP. (A) Practical and chemoselective arene reduction is an unsolved challenge in organic synthesis. (B) Case study with challenging chemoselectivity. rAP gave clean arene reduction without neccesitating special additives, whereas DC electrolysis under the identical conditions resulted in decomposition (Y-axis of the crude NMRs was adjusted to the same scaling).
The canonical Birch reduction is typically applicable to only a limited number of heterocycles. The lack of chemoselectivity of the process is evident in the absence of a literature precedent for reduction of a trivial heterocycle methyl 2-thiophenecarboxylate 1 (Figure 1A). This is understandable since Birch reduction of thiophenecarboxylic acid poses an issue of ring fragmentation. ${ }^{18}$ The ester functionality could cause more chemoselectivity issues such as overreduction or further facilitating $\mathrm{C}-\mathrm{S}$ bond cleavage. Accordingly, the reduction product 2 was previously accessed by careful Birch reduction of thiophene-2-carboxylate $\mathrm{Li}$ salt, followed by mild esterification. ${ }^{19}$

Intrigued by the absence of such an example, several representative Birch reduction conditions were applied on this simple substrate 1 (Figure 1B). Not surprisingly, standard Birch conditions ${ }^{5}$ (entry 1) resulted in complete decomposition of the starting material. The most recent modification ${ }^{16}$ (entry 2) showed attenuated reactivity, yet extensive decomposition was still observed. Donohoe's LiDBB method ${ }^{20}$ (entry 3) afforded the product in $39 \%$ yield, which is reasonable considering that this method is particularly suitable for electron-deficient heterocycles. Yet, concerns remain; LiDBB is highly sensitive (necessitating stringent degassing) and removal of large amount of DBB is problematic. Additionally, in this particular case the cost of DBB (\$915/mol, TCI) surpasses the cost of the starting material itself $(\$ 571 / \mathrm{mol}, \mathrm{TCI})$. Turning to electrochemical conditions, LER ${ }^{10}$ (entry 4) also led to decomposition. Although simple electrolysis of $\mathbf{1}$ under DC current in THF/EtOH with RVC electrodes resulted in decomposition (entry 5), it actually showed some peaks in the crude NMR indicative of trace dearomatization products. In striking contrast, under otherwise identical conditions, simply changing the waveform to rapid alternating polarity (rAP, entry 6) afforded $83 \%$ of the desired product (crude NMR shown in Figure 1). 
With the above promising results in hand, optimization was pursued using a less reactive substrate $\mathbf{3}$ as shown in Table 1. In the initial attempt, a diminished yield (29\%) was observed due to the higher aromaticity of this compound compared to $\mathbf{1}$, along with the formation of benzyl alcohol $(\mathrm{BnOH})$ presumably formed via carbonyl reduction. Standard Na or Li-based chemical conditions as well as LER on $\mathbf{3}$ did not afford any 1,4-diene product 4 (see SI). Instead, various over-reduced products and fragmentation-derived products predominated. A systematic study of chemical and electrochemical parameters revealed that dimethylsulfide (DMS) could be employed as a sacrificial electron-donor to improve the yield (Table 1, Panel A). Thus, the rest of the optimization was conducted using 3.0 equiv of DMS. Electrodes and electrolytes were found critical to achieve good reactivity (Table 1, Panel B). In particular, all electrodes examined resulted in little or no conversion with the exception of reticulated vitreous carbon (RVC) and boron-doped diamond (BDD). Due to the inexpensive nature of RVC, it was used exclusively in this study. ${ }^{21} \mathrm{~A}$ screening of electrolytes indicated that tetraalkyl ammonium salts were superior (except for the highly basic $\mathrm{Me} \mathrm{N}_{4} \cdot \mathrm{OH}$ ), while employing $\mathrm{LiClO}_{4}$ reduced conversion (Table 1, Panel C). As demonstrated in our previous rAP study, ${ }^{22}$ the electrochemical driving force delivered is a function of both current and frequency (Table 1, Panel D). Thus, either decreasing pulse width (increasing frequency) or reducing current resulted in decreased conversion. Constant potential rAP (using the same terminal potential observed under constant current with 5 equiv. of $\mathrm{Me}_{4} \mathrm{~N} \cdot \mathrm{BF}_{4}$ as electrolyte) gave a similar product distribution to constant current rAP. Since a slight warming of the reaction was noted (from $23{ }^{\circ} \mathrm{C}$ to 35 ${ }^{\circ} \mathrm{C}$, see SI) submerging the reaction vessel in a $0{ }^{\circ} \mathrm{C}$ ice bath further increased the desired product yield to $59 \%$ (best yield) by suppressing $\mathrm{BnOH}$ formation originated from the carbonyl reduction. The nature of solvent and $\mathrm{pH}$ of the reaction (Table 1, Panel E) also affected the ratio of the arene reduction vs the carbonyl reduction. Lastly, several control experiments under direct current conditions were performed to provide a comparison with the rAP-based method (Table 1, Panel F). Although a small amount of the desired product was obtained at various current densities, the yield as well as the extent of carbonyl reduction were by no means similar to the rAP-based method. In an effort to mimic the unique reactivity of rAP, constant potential experiments were attempted. Thus, the reaction potential was set to $-2.3 \mathrm{~V}$ (see SI for cyclic voltammogram of $\mathbf{3}$ ) to achieve cleanest reduction possible. Despite this effort, the yield of $\mathbf{4}$ was still far below the rAP-based method, supportive of the documented fundamental reactivity difference between rAP and DC in other studies.22-24

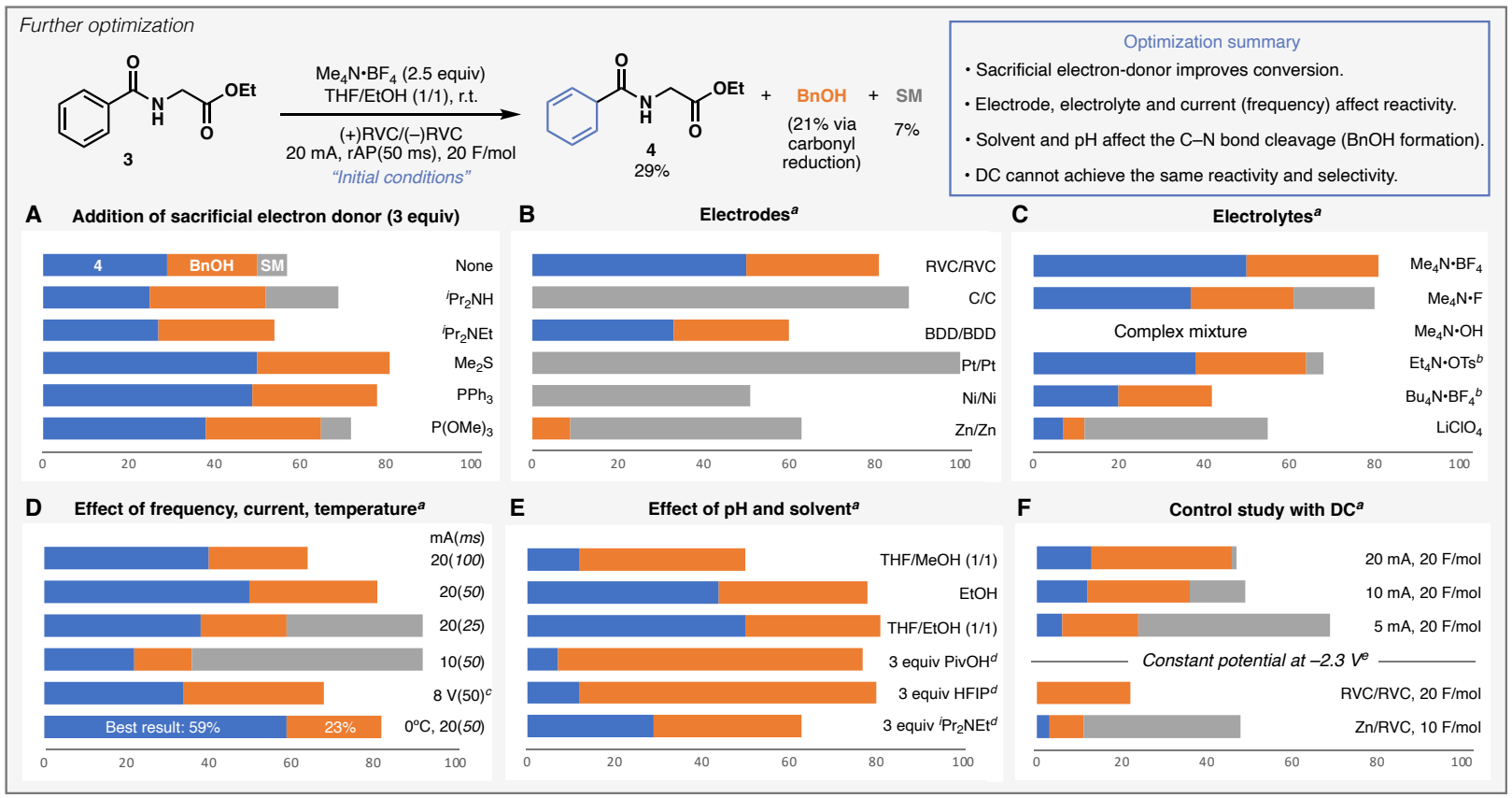

Table 1. Optimization study. All the reactions were performed on $0.1 \mathrm{mmol}$ scale under the initial conditions with a deviation indicated. Product distribution was analyzed by ${ }^{1} \mathrm{H}$ NMR. ${ }^{a}$ Reactions were performed with 3 equiv of $\mathrm{Me}_{2} \mathrm{~S}$. ${ }^{b} 1$ equiv of electrolyte was used. ${ }^{c} 5$ equiv of $\mathrm{Me}_{4} \mathrm{~N} \cdot \mathrm{BF}_{4}$ was used. ${ }^{d}$ Indicated additive was added to THF/EtOH $(1 / 1) .{ }^{e} \mathrm{Bu}_{4} \mathrm{~N} \cdot \mathrm{BF} 4(0.2 \mathrm{M})$ was used as an electrolyte. 
Table 2 demonstrates the reaction generality. Various (hetero)arenes can be reduced chemoselectively under operationally simple conditions without the need for any expensive reagents or additives (Table 2A). Complementary to standard Birch reduction, rAP reduction is most suitable for electron-deficient (hetero)arenes. The success of the reaction is predictable based on the reduction potential of an arene and a functional group that can be easily measured using CV (empirical guidance for FG tolerance is summarized in Table 2A). Thus, synthetically useful yields are obtained when a target arene has a more positive reduction potential than those of other functional groups $\left(\mathrm{E}_{\text {red,arene }}>\mathrm{E}_{\text {red,FG }}\right)$. As such, electron-deficient heteroarenes exhibit the broadest functional group tolerance with ester $(\mathbf{5 - 1 0}, \mathbf{1 2}, \mathbf{1 4 - 1 6})$, nitrile (7), allyl group (11), epoxide (8) and even alkyl chloride (13). Electron-deficient arenes are slightly less reducible than electron-deficient heterocycles, and the functional group compatibility of this class is more limited. Nevertheless, synthetically useful handles such as allyl (21), alkyne (22), ester $(\mathbf{2 3}, \mathbf{2 4}, \mathbf{2 7})$, boronate ester (25), alkyl chloride (26) and nitrile $(\mathbf{2 7}, \mathbf{2 8})$ were well-

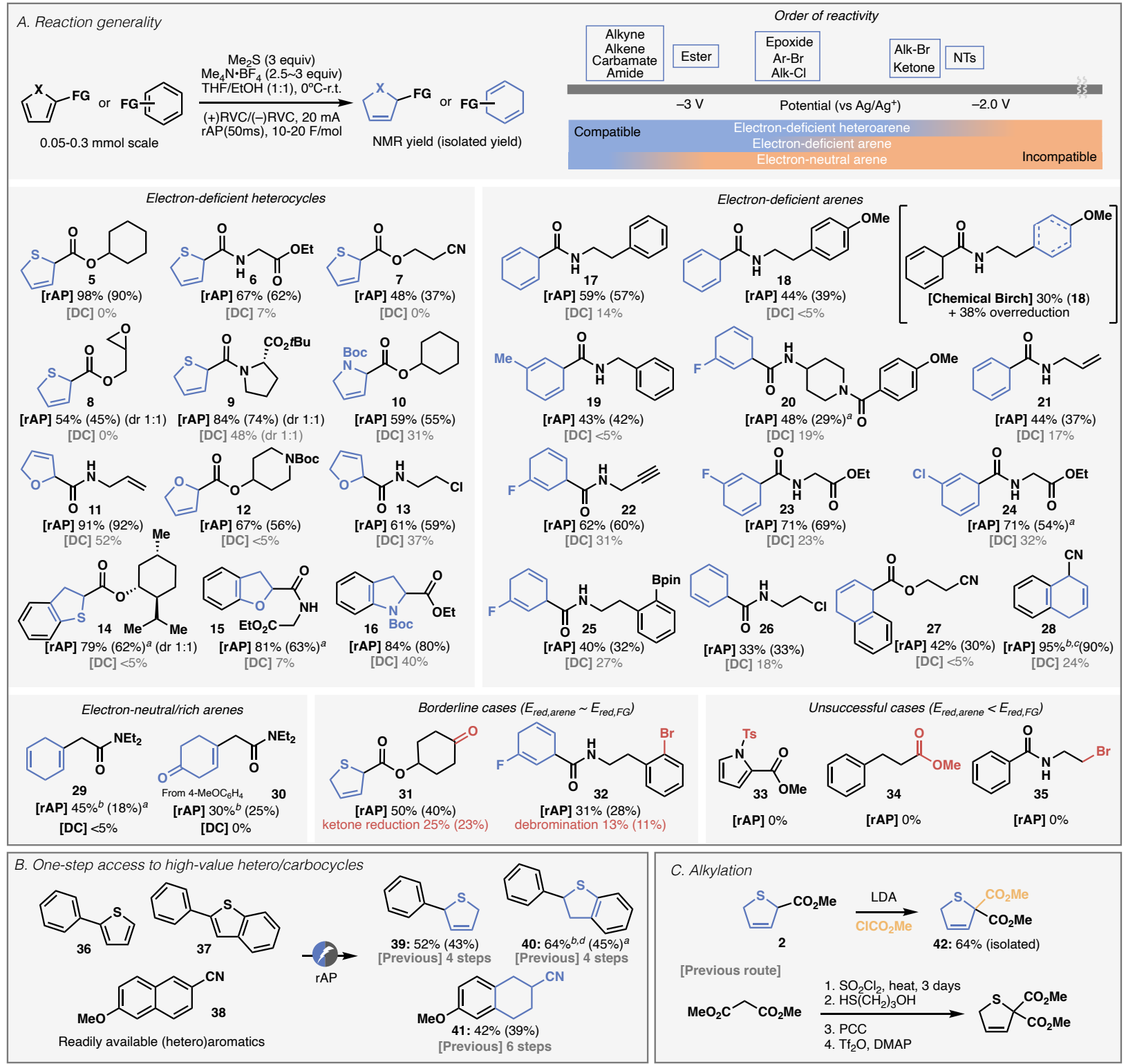

Table 2. Chemoselective reduction of (hetero)arenes by rAP. (A) Reaction generality and empirical guide for functional group tolerance. (B) Access to high-value (hetero)cyclic systems from readily available (hetero)arenes. See SI for individual reaction conditions. (C) Concise access to dihydrothiophene $\mathbf{4 2}$ via alkylation of $2 .{ }^{a}$ Large drop of isolated yield was due to purification loss. ${ }^{b} 100 \mathrm{~ms}$ pulse was used instead of $50 \mathrm{~ms}$. ${ }^{c}$ The reaction was performed in $\mathrm{MeOH}$ with $\mathrm{PivOH}$ as an additive. ${ }^{d}$ Amount of $\mathrm{Me}_{2} \mathrm{~S}$ was 10 equiv. 
tolerated. A halogen atom directly connected to an arene was also tolerated $(\mathbf{2 0}, \mathbf{2 2 - 2 5})$. Notably, differentiation of two arenes was possible as exemplified in 17-20 and 25. Achieving such chemoselectivity was found challenging under conventional Birch reduction conditions as mixture of products was obtained even if the two arenes are most electronically differentiated (Table 2A, chemical Birch for obtaining 18). Non-conjugated arenes can be reduced to afford $\mathbf{2 9}$ and $\mathbf{3 0}$ in this method, though the yields were moderate due to the low reactivity. Borderline cases also exist when $\mathrm{E}_{\text {red,arene }}$ is close to $\mathrm{E}_{\mathrm{red}, \mathrm{FG}}$, and such cases were exemplified in the reduction of 31 and 32. In accord with this simple rubric, when a functional group is more easily reducible, the method is unable to favor the reduction of (hetero)arenes (33-35).

To demonstrate the utility of this method for accessing partially saturated heterocycles, a collection of readily available heteroarenes 36-38 were enlisted (Table 2B). Subsequent alkylation could also be used to install additional complexity to an arene reduction product, which was demonstrated in the synthesis of $\mathbf{4 2}$ (Table 2C). The preparation of partially saturated heterocycles such as those exemplified above are often cumbersome since de-novo ring constructions are needed (39-42, previously required 4-6 step preparations). ${ }^{19,25-27}$ Chemoselective rAP-based arene reduction subverts conventional multi-step ring construction by repurposing existing, readily available heteroarenes.

The mechanistic details of this reaction are of great interest due to the notable reactivity difference observed between DC and rAP. A truly authoritative and complete understanding of this phenomenon would likely entail the use of various electroanalytical methods involving microelectrodes as well as rotating disc electrodes to dissect fast electron transfer kinetics under stationary and non-stationary electrolysis conditions ${ }^{28}$ Such detailed studies are beyond the scope of this Communication, and will form the basis of a separate endeavor. For the time being, several pieces of empirical evidence are presented in Figure 2 that help to rationalize the differences in bulk reactivity. For instance, a large reactivity difference was observed in the reduction of compound $\mathbf{4 3}$ between DC and rAP. Remarkably, the reduction potential of this compound $(<-3 \mathrm{~V})^{29}$ resides far outside the reduction potential of the reaction solvent (Figure 2A, solvent CV), yet rAP delivered the product in $45 \%$ NMR yield. In contrast, under otherwise identical conditions employing a standard DC waveform resulted in mostly recovered starting material. The most notable visual difference between these two reactions was gas evolution. With DC, gas evolution was clearly visible from the cathode, whereas this phenomenon was not apparent in the rAP experiment. Consistent with this observation, gas evolution from the working electrode was also confirmed after measuring CV of the solvent. We hypothesized that hydrogen gas was being formed on the cathode (reductive) in the protic medium. To prove the existence of $\mathrm{H}_{2}$ qualitatively, simple hydrogenation experiments were performed; namely, either DC or rAP was applied to the reaction solvent including cyclooctene in the presence of $\mathrm{Pd} / \mathrm{C}$ catalyst (Figure 2B). To be sure, cyclooctene is unreactive under the arene reduction conditions, and redox perturbation of $\mathrm{Pd} / \mathrm{C}$ catalyst by electrodes is unlikely since $\mathrm{Pd} / \mathrm{C}$ does not dissolve in the solvent. A considerable quantity of hydrogenated product $\mathbf{4 5}$ was observed in the DC electrolysis, while $\mathbf{4 5}$ was below the GC/MS detection limit in the rAP experiment, suggesting that $\mathrm{H}_{2}$ gas was not generated to an appreciable extent. As another proof of $\mathrm{H}_{2}$ evolution, the basicity of the reaction was probed; if proton reduction took place, a basic environment would result and facilitate base-mediated side reactions. The reduction of $\mathbf{1}$ was therefore interrupted to study a possible side reaction occurring on base-sensitive methyl ester. Indeed, transesterification product 46 was observed under DC conditions, whereas no such product was observed under rAP conditions. Consistent with these findings are literature reports of $\mathrm{CO}_{2}$ or $\mathrm{CO}$ reduction using pulsed potential instead of DC that produce less $\mathrm{H}_{2} \cdot{ }^{30-35}$ Collectively, these experiments support the mechanism summarized in Figure 2C. During the cathodic phase, (hetero)arene reduction is taking place through direct SET. This mechanism is supported by the fact that the regioselectivity of 1,4-diene products corresponds to those in conventional Birch reduction products. Regarding chemoselectivity, it follows the reduction potential of the (hetero)arene and those of existing functionalities. Proton reduction, a pathway that normally competes to diminish reactivity towards arene reduction, is largely suppressed by applying rAP. In other words, cathodic limit of solvent electrochemical window is kinetically expanded by rAP. This effect also explains improved chemoselectivity under rAP conditions by removing side reactions promoted under a highly basic environment. During the anodic phase, DMS is presumably oxidized to generate $\alpha$-ethoxy derivative. Analogous species $\mathbf{4 7}$ was detected in GC analysis 
of crude reaction mixture when heavier $\mathrm{Et}_{2} \mathrm{~S}$ was used instead of DMS. A small amount of solvent oxidation may also take place during the anodic phase.

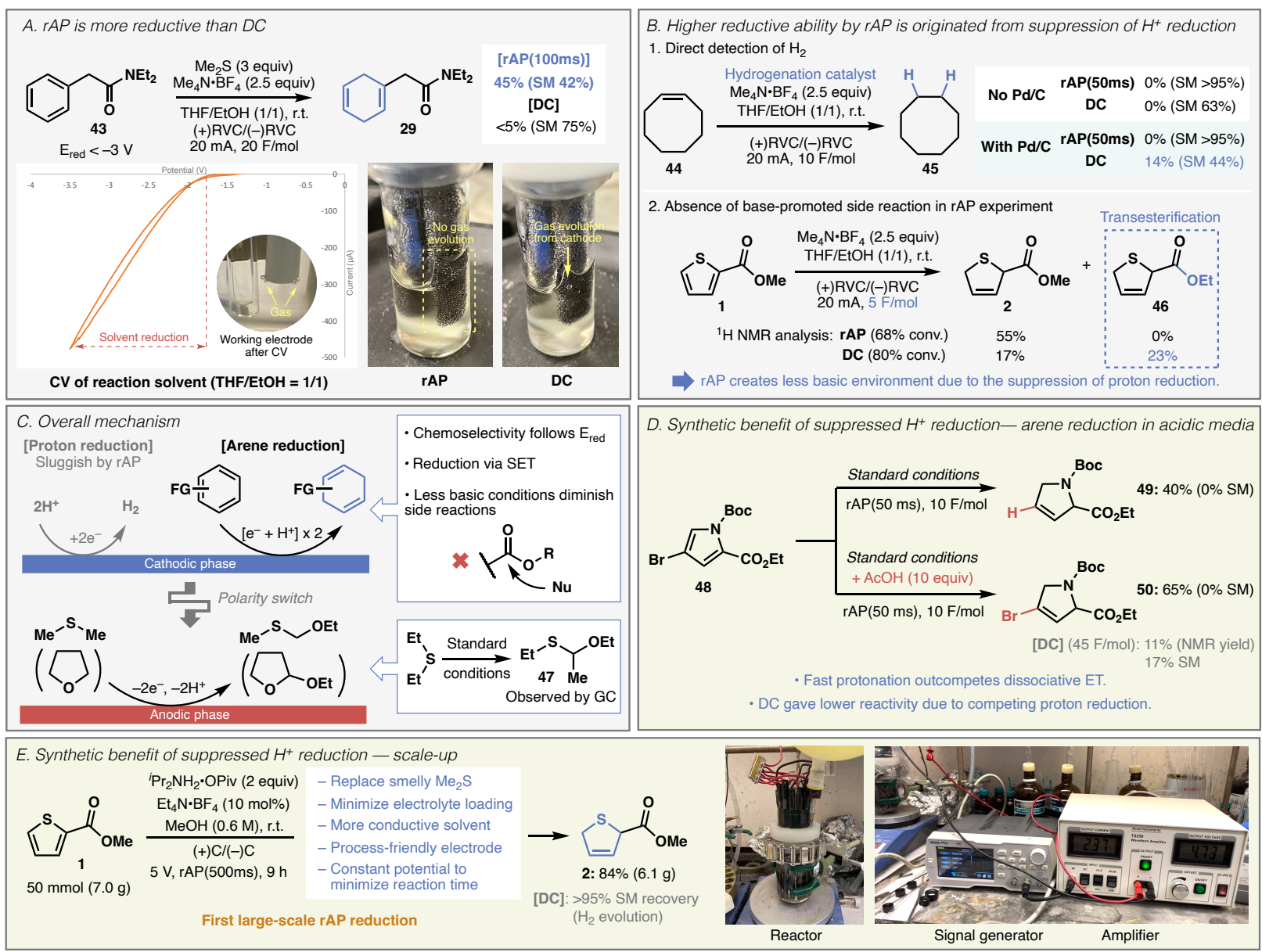

Figure 2. Mechanistic studies and synthetic advantage of outcompeting proton reduction. (A) Gas evolution was notable during DC electrolysis (including CV), whereas little gas evolution was observed with rAP. (B) Direct detection of $\mathrm{H}_{2}$ gas and resulting $\mathrm{pH}$ change in the reaction probed by transesterification. (C) Overview of cathodic and anodic reactions. (D) rAP enables efficient arene reduction in the presence of acid with unique chemoselectivity, whereas DC electrolysis suffers proton reduction. (E) First example of large-scale electrolysis with rAP. Corresponding DC reduction resulted in complete recovery of the starting material due to the competing $\mathrm{H}_{2}$ evolution.

The suppression of proton reduction can enable new types of chemoselective (hetero)arene reduction by running reactions deliberately under acidic conditions (Figure 2D). This is challenging for DC electrolysis since proton reduction is more pronounced under acidic conditions, and not advisable for chemical arene reduction owing to dangerously aggressive reactions of alkali metals with acids. As a proof of concept for this interesting possibility, standard rAP reduction of $\mathbf{4 8}$ furnished debrominated dihydropyrolidine $\mathbf{4 9}$ in $40 \%$ yield via dissociative electron-transfer, common phenomenon during single-electron reduction of halogenated arenes. In striking contrast, running the same reaction in the presence of 10 equiv of $\mathrm{AcOH}$ furnished dihydropyrrolidine $\mathbf{5 0}$ in $65 \%$ yield, maintaining the useful $\mathrm{C}-\mathrm{Br}$ bond. As expected, the corresponding DC electrolysis under identical conditions required a much longer reaction time ( $6 \mathrm{~h}, 45$ "equiv" of electrons) due to the competing proton reduction, and only delivered $11 \%$ of $\mathbf{5 0}$ along with $17 \%$ recovered $\mathbf{4 8}$. Another dramatic benefit for suppressing proton reduction was found during scale-up efforts (Figure 2E). Thus, optimized conditions for small scale could be modified to realize the reaction under more process-friendly conditions. Key modifications included replacing the sacrificial electron-donor DMS to ${ }^{i} \mathrm{Pr}_{2} \mathrm{NH}$ (buffered with pivalic acid) as well as switching solvents from $\mathrm{THF} / \mathrm{EtOH}$ to more conductive $\mathrm{MeOH}$. In addition, RVC electrodes were replaced with graphite electrodes due 
to unsatisfactory mechanical properties of RVC on scale. With these modifications, rAP-based conditions successfully afforded the product $\mathbf{2}$ in $84 \%$ isolated yield on $50 \mathrm{mmol}$ scale, whereas DC electrolysis resulted in full recovery of 1 under identical reaction conditions. ${ }^{36}$ Again, a large volume of gas evolution was observed during DC electrolysis, rendering the arene reduction unfavorable under such simple conditions.

This study demonstrates another compelling example of how the outcome of an electrochemical transformation can be completely altered simply by changing the waveform of current delivery. This time, chemoselective (hetero)arene reduction under rAP is shown to complement the scope of conventional chemical or electrochemical arene reductions. The enhanced reactivity as well as higher chemoselectivity can be explained by a suppression of competing proton reduction by the easily accessible rAP waveform. On preparative scales, no specialized equipment or engineering is required. On larger scales, a simple signal amplifier and signal generator can be employed (see SI). Although further in-depth analysis of the mechanism is necessary, the implications of this unique mode of reaction control may hold great promise for other types of chemoselective reductions of import to organic synthesis. Efficient reductive processes in protic media by rAP may also imply an interesting innovation opportunity in the areas of electrochemical $\mathrm{CO}_{2}$ reduction as well as nitrogen fixation, where proton reduction often competes.

\section{ASSOCIATED CONTENT}

\section{Supporting Information}

Experimental procedures, X-ray, cyclic voltammogram and characterizations of compounds.

\section{AUTHOR INFORMATION}

\section{Corresponding Author}

*yukawama@scripps.edu

*pbaran@scripps.edu

\section{Author Contributions}

YK and PSB conceptualized the study, KH, YK, PSB designed experiments and KH, YK performed experiments. All the authors contributed to data analysis and manuscript preparation.

\section{Notes}

Authors declare no competing financial interest.

\section{ACKNOWLEDGMENT}

Financial support for this work was provided by National Science Foundation Center for Synthetic Organic Electrochemistry (CHE-2002158), and the National Institutes of Health (grant number GM-118176). We are grateful to D.-H. Huang and L. Pasternack (Scripps Research) for NMR spectroscopic assistance, and M. Gembicky (UCSD) for X-ray analysis. We also thank A. F. Garrido-Castro, M.D. Palkowitz and S. B. J. Kan for helpful discussions.

\section{REFERENCES}

(1) Huck, C. J.; Sarlah, D. Shaping Molecular Landscapes: Recent Advances, Opportunities, and Challenges in Dearomatization. Chem 2020, 6, 1589-1603.

(2) Birch, A. J. The Birch Reduction in Organic Synthesis. Pure Appl. Chem. 1996, 68, 553-556. (3) Donohoe, T. J.; Garg, R.; Stevenson, C. A. Prospects for Stereocontrol in the Reduction of Aromatic Compounds. Tetrahedron Asymmetry 1996, 7, 317-344.

(4) Hook, J. M.; Mander, L. N. Recent Developments in the Birch Reduction of Aromatic Compounds: Applications to the Synthesis of Natural Products. Nat. Prod.Rep. 1986, 3, 35-85.

(5) Rabideau, P. W.; Marcinow, Z. Organic React. 1992, 42, 1-334.

(6) Dye, J. L.; Cram, K. D.; Urbin, S. A.; Redko, M. Y.; Jackson, J. E.; Lefenfeld, M. Alkali Metals Plus Silica Gel: Powerful Reducing Agents and Convenient Hydrogen Sources. J. Am. Chem. Soc. 2005, 127, 9338-9339. 
(7) Nandi, P.; Redko, M. Y.; Petersen, K.; Dye, J. L.; Lefenfeld, M.; Vogt, P. F.; Jackson, J. E. Alkali Metals in Silica Gel (M-SG): A New Reagent for Desulfonation of Amines. Org. Lett. 2008, 10, 5441-5444.

(8) Costanzo, M. J.; Patel, M. N.; Petersen, K. A.; Vogt, P.F. Ammonia-Free Birch Reductions with Sodium Stabilized in Silica Gel, Na-SG(I). Tetrahedron Lett. 2009, 50, 5463-5466.

(9) Lei, P.; Ding, Y.; Zhang, X.; Adijiang, A.; Li, H.; Ling, Y.; An, J. A Practical and Chemoselective Ammonia-Free Birch Reduction. Org. Lett. 2018, 20,3439-3442.

(10) Peters, B. K.; Rodriguez, K. X.; Reisberg, S. H.; Beil, S. B.; Hickey, D. P.; Kawamata, Y.; Collins, M.; Starr, J.; Chen, L.; Udyavara, S.; Klunder, K.; Gorey, T. J.; Anderson, S. L.; Neurock, M.; Minteer, S. D.; Baran, P. S. Scalable and Safe Synthetic Organic Electroreduction Inspired by Li-Ion Battery Chemistry. Science 2019, 363, 838-845.

(11) Cole, J. P.; Chen, D.-F.; Kudisch, M.; Pearson, R. M.; Lim, C.-H.; Miyake, G. M. Organocatalyzed Birch Reduction Driven by Visible Light. J. Am. Chem. Soc. 2020, 142, 13573-13581.

(12) Chatterjee, A.; König, B. Birch-Type Photoreduction of Arenes and Heteroarenes by Sensitized Electron Transfer. Angew. Chem. Int. Ed. 2019, 58, 14289-14294.

(13) Benkeser, R. A.; Robinson, R. E.; Sauve, D. M.; Thomas, O. H. Reduction of Organic Compounds by Lithium in Low Molecular Weight Amines. I. Selective Reduction of Aromatic Hydrocarbons to Monoölefins. J. Am. Chem. Soc. 1955, 77, 3230-3233.

(14) Benkeser, R. A.; Arnold, C.; Lambert, R. F.; Thomas, O. H. Reduction of Organic Compounds by Lithium in Low Molecular Weight Amines. III. Reduction of Aromatic Compounds Containing Functional Groups. J. Am. Chem. Soc. 1955, 77, 6042-6045.

(15) Benkeser, R. A.; Agnihotri, R. K.; Burrous, M. L.; Kaiser, E. M.; Mallan, J. M.; Ryan, P. W. Highly Selective Lithium-Amine Reducing Systems. The Selective Reduction of Aromatic Compounds by Lithium in Mixed Amine Solvents. J. Org. Chem. 1964, 29, 1313-1316.

(16) Burrows, J.; Kamo, S.; Koide, K. Scalable Birch Reduction with Lithium and Ethylenediamine in Tetrahydrofuran. Science 2021, 374, 741-746.

(17) Donohoe, T. J.; Thomas, R. E. Partial Reduction of Pyrroles: Application to Natural Product Synthesis. Chem. Rec. 2007, 7, 180-190.

(18) Blenderman, W. G.; Joullié, M. M.; Preti, G. The Birch Reduction of Thiophine-2-Carboxylic Acid. Tetrahedron Lett. 1979, 20, 4985-4988.

(19) Altenbach, H.-J.; Brauer, D. J.; Merhof, G. F. Synthesis of 1-Deoxy-4-Thio-D-Ribose Starting from Thiophene2-Carboxylic Acid. Tetrahedron 1997, 53, 6019-6026.

(20) Donohoe, T. J.; House, D. Ammonia Free Partial Reduction of Aromatic Compounds Using Lithium Di-tertButylbiphenyl (LiDBB). J. Org. Chem. 2002, 67, 5015-5018.

(21) Although RVC electrodes were exclusively used in small-scale reactions, graphite electrodes were also found effective as demonstrated in $50 \mathrm{mmol}$ scale reaction in Figure 2E.

(22) Kawamata, Y.; Hayashi, K.; Carlson, E.; Shaji, S.; Waldmann, D.; Simmons, B. J.; Edwards, J. T.; Zapf, C. W.; Saito, M.; Baran, P. S. Chemoselective Electrosynthesis Using Rapid Alternating Polarity. J. Am. Chem. Soc. 2021, $143,16580-16588$.

(23) Rodrigo, S.; Um, C.; Mixdorf, J. C.; Gunasekera, D.; Nguyen, H. M.; Luo, L. Alternating Current Electrolysis for Organic Electrosynthesis: Trifluoromethylation of (Hetero)Arenes. Org. Lett. 2020, 22, 6719-6723.

(24) Rodorigo, S.; D, G.; Mahajan, J. P.; Luo, L. Alternating Current Electrolysis for Organic Synthesis. Current Opinion in Electrochemistry 2021, 28, 100712.

(25) Chatterjee, A.; Roy, D.; Chatterjee, S. An Efficient General Method for the Synthesis of Some Intermediates for Heterocyclic Steroids. Synthesis 1981, 1981, 449-451.

(26) Liao, Q.; You, W.; Lou, Z.-B.; Wen, L.-R.; Xi, C. Copper-Catalyzed Tandem S-Alkylation and S-Alkenylation of Sodium Sulfide: Synthesis of 2,3-Dihydrothiophenes and Thiophenes. Tetrahedron Lett. 2013, 54, 1475-1477.

(27) Rogers, E.; Araki, H.; Batory, L. A.; McInnis, C. E.; Njardarson, J. T. Highly Selective Copper-Catalyzed Ring Expansion of Vinyl Thiiranes: Application to Synthesis of Biotin and the Heterocyclic Core of Plavix. J. Am. Chem. Soc. 2007, 129, 2768-2769.

(28) Bamford, C. H.; Tipper, C. F. H.; Compton, R. G. Electrode Kinetics: Principles and Methodology; Elsevier, 1986.

(29) Mortensen, J.; Heinze, J. The Electrochemical Reduction of Benzene-First Direct Determination of the Reduction Potential. Angew. Chem. Int. Ed. 1984, 23, 84-85.

(30) Kimura, K. W.; Fritz, K. E.; Kim, J.; Suntivich, J.; Abruña, H. D.; Hanrath, T. Controlled Selectivity of $\mathrm{CO}_{2}$ Reduction on Copper by Pulsing the Electrochemical Potential. Chemsuschem 2018, 11 (11), 1781-1786.

(31) Kim, C.; Weng, L.-C.; Bell, A. T. Impact of Pulsed Electrochemical Reduction of $\mathrm{CO}_{2}$ on the Formation of C2+ Products over Cu. ACS Catal. 2020, 10, 12403-12413. 
(32) Strain, J. M.; Gulati, S.; Pishgar, S.; Spurgeon, J. M. Pulsed Electrochemical Carbon Monoxide Reduction on Oxide-Derived Copper Catalyst. Chemsuschem 2020, 13, 3028-3033.

(33) Casebolt, R.; Levine, K.; Suntivich, J.; Hanrath, T. Pulse Check: Potential Opportunities in Pulsed Electrochemical $\mathrm{CO}_{2}$ Reduction. Joule 2021, 5, 1987-2026.

(34) Casebolt, R.; Kimura, K. W.; Levine, K.; DaSilva, J. A. C.; Kim, J.; Dunbar, T. A.; Suntivich, J.; Hanrath, T. Effect of Electrolyte Composition and Concentration on Pulsed Potential Electrochemical $\mathrm{CO}_{2}$ Reduction. Chemelectrochem 2021, 8, 681-688.

(35) Tang, Z.; Nishiwaki, E.; Fritz, K. E.; Hanrath, T.; Suntivich, J. Cu(I) Reducibility Controls Ethylene vs Ethanol Selectivity on (100)-Textured Copper during Pulsed $\mathrm{CO}_{2}$ Reduction. ACS Appl. Mater. Interfaces 2021, 13, 1405014055 .

(36) The DC electrolysis comparison was carried out on $2.0 \mathrm{mmol}$ scale under otherwise identical reaction conditions and substrate concentration. 